DESY 04-115

hep-ph/0407045

June 2004

\title{
NNLO coefficient functions of Higgs and Drell-Yan cross sections in Mellin space
}

\author{
J. Blümlein ${ }^{\mathrm{a}}$ and V. Ravindran ${ }^{\mathrm{a}, \mathrm{b} *}$ \\ a Deutsches Elektronen Synchrotron, DESY, Platanenallee 6, D-15738 Zeuthen, Germany. \\ ${ }^{\mathrm{b}}$ Harish-Chandra Research Institute, Chhatnag Road, Jhunsi, Allahabad, India.
}

We calculate the Mellin moments of next-to-next-to-leading order coefficient functions of the Drell-Yan and Higgs production cross sections. The results can be expressed in term of finite harmonic sums which are maximally threefold up to weight four. Various algebraic relations among these finite sums reduce the complexity of the results suitable for fast numerical evaluations. It is shown that only five non-trivial functions occur besides Euler's $\psi$-function in the representation of these Wilson coefficients.

\section{Introduction}

With the present $p p$-collider Tevatron [1 and the upcoming large hadron collider (LHC) [2] at CERN, the need for precise predictions from theory have become more and more important. The Drell-Yan (DY) cross section which is known upto the NNLO level 3 not only tests the reliability of perturbative QCD (pQCD) but also reduces the uncertainties coming from theory in order to make background studies more reliable for new particle searches and physics beyond the Standard Model. Similarly, higher order corrections to the total cross section [4 for the Higgs boson production make the predictions more reliable for phenomenological studies. In the following we will study the structure of these corrections in the Mellin space using various algebraic identities relating the resulting finite harmonic sums. This representation allows a considerable reduction of the set of basic functions needed to represent the 2-loop Wilson coefficients.

\footnotetext{
*Based on the talk given at 7th DESY Workshop on Elementary Particle Theory, Loops and Legs in Quantum Field Theory, April 25 -30, 2004, Zinnowitz (Usedom Island), Germany
}

\section{Coefficient Functions}

Due to mass factorization, hadronic cross sections such as for the DY-process and Higgsboson production can be expressed in terms of Mellin convolutions of the perturbatively computable coefficient functions $\Delta_{a b}\left(x, Q^{2}, \mu^{2}\right)$ and non-perturbative parton distributions, $f_{a}\left(x, \mu^{2}\right)$, of incoming hadrons.

$$
\begin{aligned}
\sigma\left(x, Q^{2}\right)= & \int_{x}^{1} \frac{d x_{1}}{x_{1}} \int_{\frac{x}{x_{1}}} \frac{d x_{2}}{x_{2}} f_{a}\left(x_{1}, \mu^{2}\right) f_{b}\left(x_{2}, \mu^{2}\right) \\
& \times \Delta_{a b}\left(\frac{x}{x_{1} x_{2}}, \frac{Q^{2}}{\mu^{2}}\right) .
\end{aligned}
$$

Though the parton densities are not calculable in the $\mathrm{pQCD}$, their evolution with respect to a scale is computable, thanks to the renormalization group (RG) equations governing mass factorization. Hence higher order corrections to hadronic reactions enter through two sources viz, the coefficient functions and the RG-equations of parton distribution functions. In this paper we will concentrate only on the coefficient functions. Details of the calculation will be given in [5].

The perturbatively calculable coefficient functions are usually computed in terms of the scaling 
variable $x=Q^{2} / s$ where $s$ is the center of mass energy of the incoming partonic system. $Q^{2}$ is the invariant mass of the final di-lepton pair (for DY) or mass squared of the Higgs boson. The results are expressible in terms of polynomials in $x$, logarithms and Nielsen integrals [6] defined by

$S_{n, p}(x)=\frac{(-1)^{n+p-1}}{(n-1) ! p !} \int_{0}^{1} \frac{d z}{z} \log ^{n-1}(z) \log ^{p}(1-z x)$

In practice, using (1), one performs the integration over $x_{1}$ and $x_{2}$ after folding the perturbatively computed coefficient functions with the appropriate parton distributions. This involves further evaluation of various Nielsen integrals given in (2) or even more general functions. Due to this the complexity of the numerical evaluation of the hadronic cross sections is rather large. In the following, we will present an alternative treatment of the evaluation of the total cross section upto NNLO level by working in Mellin space. Such techniques have been used in the past to compute deep inelastic scattering cross sections and indeed they are found to be most suitable for various resummation programs [7. Recently, similar work has also been performed for the Wilson coefficients for polarized and unpolarized deeply inelastic scattering [89].

\section{Mellin Moments and Finite Harmonic Sums}

Using the Mellin transform [10] of a given function $F(x)$

$M[F](N)=\int_{0}^{1} d x x^{N-1} F(x)$

the cross section (1) in the Mellin space ( $N$-space) becomes

$$
\begin{aligned}
M[\sigma]\left(N, Q^{2}\right)= & M\left[f_{a}\right]\left(N, \mu^{2}\right) M\left[f_{b}\right]\left(N, \mu^{2}\right) \\
& \times M\left[\Delta_{a b}\right]\left(N, \frac{Q^{2}}{\mu^{2}}\right) .
\end{aligned}
$$

As is clear from the above equation, the convolutions of functions in $x$-space reduce to simple products of Mellin moments in $N$-space. The Mellin moments of these functions can be analytically continued [11] to complex values of $N$ so that one can use various analyticity properties of these functions in the complex $N$-plane to evaluate them efficiently.

For our analysis, the starting point is eqn. (1) with given parton densities $f_{a}\left(x, \mu^{2}\right)$ and known coefficient functions $\Delta_{a b}\left(x, Q^{2}\right)$ computed upto NNLO in pQCD. We then compute the Mellin moments of these functions in $N$-space and perform their analytic continuation to complex values of $N$. At the end, we substitute them back into eqn. (4) and perform the inverse Mellin transformation to arrive at the results in $x$-space using a suitable contour in the complex $N$-plane. Compared to the direct numerical convolution in $x$-space the numerical computation using the $N$ space results is much faster.

Since we are dealing with the DY/Higgs total production cross sections, they depend only on two variables, the scaling variable $x$ and the virtuality $Q^{2}$, the invariant mass of the final state. The set of functions contributing to the two-loop coefficient functions in $x$ space contains about 80 elements. Their representation in Mellin space has been given in [12] in terms of a specific class of sums 1213 14 15, the finite harmonic sums.

The finite harmonic sum of $m$-indices is defined as

$$
\begin{aligned}
S_{k_{1} \ldots k_{m}}(N)= & \sum_{n_{1}=1}^{N} \frac{\left[\operatorname{sign}\left(k_{1}\right)\right]^{n_{1}}}{n_{1}^{\left|k_{1}\right|}} \sum_{n_{2}=1}^{n_{1}} \frac{\left[\operatorname{sign}\left(k_{2}\right)\right]^{n_{2}}}{n_{2}^{\left|k_{2}\right|}} \\
& \cdots \sum_{n_{m}=1}^{n_{m}-1} \frac{\left[\operatorname{sign}\left(k_{m}\right)\right]^{n_{m}}}{n_{m}^{\left|k_{m}\right|}}
\end{aligned}
$$

with $l, k_{l} \neq 0$. For example,

$$
\begin{aligned}
& M\left[\left(\frac{\log ^{3}(1-x)}{(1-x)}\right)_{+}\right](N)=\frac{1}{4} S_{1}^{4}(N-1) \\
& +\frac{3}{2} S_{1}^{2}(N-1) S_{2}(N-1)+\frac{3}{4} S_{2}^{2}(N-1) \\
& +2 S_{1}(N-1) S_{3}(N-1)+\frac{3}{2} S_{4}(N-1) .
\end{aligned}
$$

Here the right hand side is a polynomial out of only single harmonic sums. Similarly, a combination of various Nielsen integrals may have a simple 
structure in $N$-space 12 :

$$
\begin{aligned}
& M\left[S_{12}(-x)+\frac{1}{2}\left(2 \mathcal{L} i_{2}(-x) \log (1+x)+\log (x)\right.\right. \\
& \left.\left.\times \log ^{2}(1+x)\right)\right](N)=\frac{1}{N}\left(-\frac{1}{2} \zeta_{2} \log (2)+\frac{1}{8} \zeta_{3}\right. \\
& -(-1)^{N}\left(S_{1,-2}(N)+\frac{1}{2} \zeta_{2}\left(S_{1}(N)-S_{-1}(N)\right)\right. \\
& \left.\left.+\frac{1}{8} \zeta_{3}-\frac{1}{2} \zeta_{2} \log (2)\right)\right)
\end{aligned}
$$

The single harmonic sums $(m=1)$ obey the following integral representation

$$
\begin{aligned}
S_{ \pm k}(N) & =\int_{0}^{1} \frac{d x_{1}}{x_{1}} \cdots \int_{0}^{x_{k-1}} \frac{\left( \pm x_{k}\right)^{N}-1}{x_{k} \mp 1} \\
& =\frac{(-1)^{k-1}}{(k-1) !} \int_{0}^{1} d x \log ^{k-1}(x) \frac{( \pm x)^{N}-1}{x \mp 1} .
\end{aligned}
$$

Using the above representation for the single sums and the following formula,

$$
\sum_{k=1}^{N} \frac{( \pm x)^{k}}{k^{l}}=\frac{(-1)^{l-1}}{(l-1) !} \int_{0}^{x} d z \log ^{l-1}(z) \frac{( \pm z)^{N}-1}{z \mp 1}
$$

one derives integral representations for the multiple sums $S_{k_{1} \ldots k_{m}}(N)$. For our purpose, we have made extensive use of the results given in [12].

\section{Algebraic Relations}

Finite harmonic sums are related to each other by various algebraic relations 16/17/12. These relations result from the shuffle product of two harmonic sums. Special cases follow from partial or complete index permutations.

The permutation relation for sums with two indices is given by

$$
\begin{aligned}
S_{m, n}(N)+S_{n, m}(N)= & S_{m}(N) S_{n}(N) \\
& +S_{m \wedge n}(N),
\end{aligned}
$$

with $m \wedge n=\operatorname{sign}\{m\} \operatorname{sign}\{n\}(|m|+|n|)$.

For harmonic sums with three indices the following identity is used to derive various relations

$$
\sum_{\text {perm }\{l, m, n\}} S_{l, m, n}(N)=S_{l}(N) S_{m}(N) S_{n}(N)
$$

$$
+\sum_{i n v} S_{\text {perm }\{l, m, n\}}(N) S_{m \wedge n}+2 S_{l \wedge m \wedge n}(11)
$$

where "inv perm" means the invariant permutations and "perm" means permutations.

$$
\begin{aligned}
S_{1,2,1}(N)= & -2 S_{2,1,1}(N)+S_{3,1}(N) \\
& +S_{1}(N) S_{2,1}(N)+S_{2,2}(N) \\
S_{1,1,2}(N)= & S_{2,1,1}(N)+\frac{1}{2}\left(S _ { 1 } ( N ) \left(S_{1,2}(N)\right.\right. \\
& \left.\left.-S_{2,1}(N)\right)+S_{1,3}(N)-S_{3,1}(N)\right) \\
S_{1,-2,1}(N)= & -2 S_{-2,1,1}(N)+S_{-3,1}(N) \\
& +S_{1}(N) S_{-2,1}(N)+S_{-2,2}(N) \\
S_{1,1,-2}(N)= & S_{-2,1,1}(N)+S_{-2}(N) S_{2}(N) \\
& -S_{-2,2}(N)-S_{-2}(N) S_{1,1}(N) \\
& +S_{1}(N) S_{1,-2}(N)+S_{1,-3}(N) \\
& -S_{1}(N) S_{-3}(N)
\end{aligned}
$$

\section{Coefficient Functions in $N$-Space}

We have computed the Mellin moments for the DY and the Higgs coefficient functions using the list given in [12] and the algebraic identities given in the previous sections. For a general investigation of these relations, see 1418. The algebraic relations considerably simply our final result. At the intermediate stages of the computation we encounter various complicated sums such as $S_{1,-1,2}$, $S_{-1,-1,-2}, S_{-1,-2,-1}, S_{-2,-1,-1}, S_{2,-1,1}, S_{1,2,-1}$, $S_{2,1-1}, S_{-1,1,2}, S_{-1,1,2}$. At the end, most of these sums disappear leaving only few sums like $S_{-2,1,1}$, $S_{2,1,1}$. We have listed below the sums that appear at the end of the computation.

We obtain eight single sums $S_{ \pm i}(N)$ with $i=$ $1 \ldots 4$,

$S_{-4}(N)=(-1)^{N+1} \frac{1}{6} M\left[\frac{\log ^{3}(x)}{1+x}\right](N+1)-\frac{7 \zeta_{2}^{2}}{20}$

$S_{-3}(N)=(-1)^{N} \frac{1}{2} M\left[\frac{\log ^{2}(x)}{1+x}\right](N+1)-\frac{3}{4} \zeta_{3}$ 


$$
\begin{aligned}
S_{-2}(N) & =(-1)^{N+1} M\left[\frac{\log (x)}{1+x}\right](N+1)-\frac{1}{2} \zeta_{2} \\
S_{-1}(N) & =(-1)^{N} M\left[\frac{1}{1+x}\right](N+1)-\log (2) \\
S_{4}(N) & =\frac{1}{6} M\left[\frac{\log ^{3}(x)}{1-x}\right](N+1)+\frac{2}{5} \zeta_{2}^{2} \\
S_{3}(N) & =-\frac{1}{2} M\left[\frac{\log ^{2}(x)}{1-x}\right](N+1)+\zeta_{3} \\
S_{2}(N) & =M\left[\frac{\log (x)}{1-x}\right](N+1)+\zeta_{2} \\
S_{1}(N) & =-M\left[\left(\frac{1}{1-x}\right)_{+}\right](N+1) .
\end{aligned}
$$

These harmonic sums can be solely expressed in terms of the Euler $\psi$-function and the $\beta$ function [17 and their derivatives, which is related to the former combining two $\psi$-functions with shifted argument. These functions represent at the same time the analytic continuation of these harmonic sums. The following five double sums $S_{-3,1}(N), S_{-2,1}(N), S_{-2,2}(N), S_{2,1}(N)$, $S_{3,1}(N)$ occur :

$$
\begin{aligned}
S_{-3,1}(N)= & (-1)^{N} M\left[\frac{\mathcal{L} i_{3}(x)}{1+x}\right](N+1) \\
& +\zeta_{2} S_{-2}(N)-\zeta_{3} S_{-1}(N)-\frac{3}{5} \zeta_{2}^{2} \\
& +2 \mathcal{L}_{4}\left(\frac{1}{2}\right)+\frac{3}{4} \zeta_{3} \log (2) \\
& -\frac{1}{2} \zeta_{2} \log ^{2}(2)+\frac{1}{12} \log ^{4}(2) \\
S_{-2,1}(N)= & -(-1)^{N} M\left[\frac{\mathcal{L} i_{2}(x)}{1+x}\right](N+1) \\
& +\zeta_{2} S_{-1}(N)-\frac{5}{8} \zeta_{3} \\
& +\zeta_{2} \log (2) \\
S_{-2,2}(N)= & -(-1)^{N} M\left[\frac { 1 } { 1 + x } \left(2 \mathcal{L} i_{3}(x)\right.\right.
\end{aligned}
$$

$$
\begin{aligned}
& \left.\left.-\log (x)\left(\mathcal{L} i_{2}(x)+\zeta_{2}\right)\right)\right](N+1) \\
& +\zeta_{2} S_{-2}(N)+2 \zeta_{3} S_{-1}(N) \\
& +\frac{71}{40} \zeta_{2}^{2}-4 \mathcal{L} i_{4}\left(\frac{1}{2}\right) \\
& -\frac{3}{2} \zeta_{3} \log (2)+\zeta_{2} \log ^{2}(2)-\frac{\log ^{4}(2)}{6} \\
S_{2,1}(N)= & M\left[\left(\frac{\mathcal{L} i_{2}(x)}{1-x}\right){ }_{+}\right](N+1)+\zeta_{2} S_{1}(N) \\
S_{3,1}(N)= & -\frac{1}{2} M\left[\frac{\mathcal{L} i_{2}(x) \log (x)}{1-x}\right](N+1) \\
& +\zeta_{2} S_{2}(N)-\frac{1}{4} S_{2}^{2}(N)-\frac{1}{4} S_{4}(N) \\
& -\frac{3}{20} \zeta_{2}^{2}
\end{aligned}
$$

Furthermore, two triple sums $S_{-2,1,1}(N)$, $S_{2,1,1}(N)$ contribute :

$$
\begin{aligned}
S_{-2,1,1}(N)= & -(-1)^{N} M\left[\frac{S_{12}(x)}{1+x}\right](N+1) \\
& +\zeta_{3} S_{-1}(N)-\mathcal{L} i_{4}\left(\frac{1}{2}\right)+\frac{1}{8} \zeta_{2}^{2} \\
& +\frac{1}{8} \zeta_{3} \log (2)+\frac{1}{4} \zeta_{2} \log ^{2}(2) \\
& -\frac{1}{24} \log ^{4}(2) \\
S_{2,1,1}(N)= & M\left[\left(\frac{S_{12}(x)}{1-x}\right)_{+}\right](N+1) \\
& +\zeta_{3} S_{1}(N)
\end{aligned}
$$

From our final expression, we observe that only very few functions do finally contribute to the Wilson coefficients. We list them below for completeness:

$$
\begin{array}{ll}
\frac{\log ^{n}(x)}{1-x} & \frac{\log ^{n}(x)}{1+x} \quad n=0,1,2,3 \\
\frac{\mathcal{L} i_{2}(x) \log ^{n}(x)}{1-x} & \frac{\mathcal{L} i_{2}(x) \log ^{n}(x)}{1+x} \quad n=0,1
\end{array}
$$




$$
\begin{array}{ll}
\frac{S_{12}(x)}{1-x} & \frac{S_{12}(x)}{1+x} \\
\frac{\mathcal{L} i_{3}(x)}{1+x} &
\end{array}
$$

Since functions weighted by a factor $\ln ^{n}(x)$ are related to the un-weighted functions in Mellin space by

$M\left[\ln ^{n}(x) f(x)\right](N)=\frac{\partial^{n}}{\partial N^{n}} M[f(x)](N)$

and single harmonic sums can be expressed by the well-known $\psi$-functions only five non-trivial functions are needed to express the two-loop Wilson coefficients for the polarized and unpolarized DY-process and the scattering cross sections of hadronic Higgs-boson and pseudoscalar Higgsboson production.

The next step is to multiply the appropriate parton densities computed in complex $N$-space with our coefficient functions obtained to invert back to $x$-space for phenomenological studies.

The Mellin inversion [19] of a function $\tilde{F}(N)$ is given by

$F(x)=\frac{1}{2 \pi i} \int_{c-i \infty}^{c+i \infty} d N x^{-N} \tilde{F}(N)$

Here the parameter $c$ is the intersection of the contour on the real axis and is chosen in such a way that the integral $\int_{0}^{1} d x x^{c-1} F(x)$ is convergent. In other words, $c$ should lie on the right of rightmost singularity of the function $\tilde{F}(N)$. The shape of the contour can be deformed at our convenience provided all singularities are covered.

\section{Conclusion}

We have systematically analyzed the mathematical structure behind the NNLO coefficient functions for DY and Higgs production using Mellin moment techniques. Use of various algebraic identities which relate the finite harmonic sums in Mellin $N$-space reduces the complexity of the results from around 80 functions to only five basic functions, the $\psi$-function and a few derivatives thereof. This is very useful not only to understand the nature of higher order corrections but also to perform fast numerical calculations at high precision for phenomenological applications and fits to data. The same structures are found in the case of polarized and unpolarized 2-loop fragmentation functions [20]. Together with the results of 8 it is now shown that these structures are in common for all known massless 2-loop Wilson coefficients.

Acknowledgment We thank S. Moch for discussions. V.R. would like to thank DESY for their kind hospitality extended to him and for a lively meeting, Loops and Legs 2004. This paper was supported in part by DFG Sonderforschungsbereich Transregio 9, Computergestützte Theoretische Physik and EU grant HPRN-CT-200000149 .

\section{REFERENCES}

1. Tevatron Electroweak Working Group, [D0 Collaboration], hep-ex/0404010

2. CMS Coll., Technical Proposal, report CERN/LHCC/94-38, ATLAS Coll., ATLAS Detector and Physics Performance: Technical Design Report, Vol.2, report CERN/LHCC/99-15 (1999).

3. T. Matsuura, S.C. van der Marck, W.L. van Neerven, Nucl. Phys. B319 (1989) 570;

R. Hamberg, W.L. van Neerven, T. Matsuura, Nucl.Phys. B359 (1991) 343;

V. Ravindran, J. Smith and W. L. van Neerven, Nucl. Phys. B682 (2004) 421.

4. S. Catani, D. de Florian, M. Grazzini, JHEP (2001) 0105:025;

R.V. Harlander, W.B. Kilgore, Phys. Rev. D64 (2001) 013015; Phys. Rev. Lett. 88 (2002) 201801; JHEP (2002) 0210:017;

C. Anastasiou, K. Melnikov, Nucl. Phys. B646 (2002) 220;

V. Ravindran, J. Smith and W. L. van Neerven, Nucl. Phys. B665 (2003) 325.

5. J. Blümlein and V. Ravindran, in preparation.

6. E. Nielsen, Nova Acta Leopold. XC (1909) 121 ;

S. Kölbig, Siam J. Math. Anal. 17 (1986) 1232 ;

L. Lewin, Dilogarithms and Associated Func- 
tions (Macdonald, London, 1958); Polylogarithms and Associated Functions (North Holland, New York, 1981).

7. J. Blümlein and A. Vogt, Phys. Lett. B370 (1996) 149; Acta Phys. Pol. B 271309 (1996);

Phys. Lett. B 386 (1996) 350; Phys. Rev. D 57 (1998) 1; D58 (1998) 014020;

J. Blümlein, S. Riemersma, and A. Vogt, Nucl. Phys. B (Proc. Suppl.) 51C (1996) 50; Eur. Phys. J.C 1 (1998) 255, J. Blümlein, V. Ravindran, W.L. van Neerven and A. Vogt, hep-ph/9806368

R.K. Ellis, Z. Kunszt, and E. Levin, Nucl. Phys. B420 (1994) 517, B433 (1995) 498(E).

8. J. Blümlein and S. Moch, in preparation.

9. J. Blümlein, these Proceedings, hep-ph/0407044

10. H. Mellin, Acta Math. 25 (1902) 139.

11. J. Blümlein, Comput. Phys. Commun. 133 (2000) 76 .

12. J. Blümlein and S. Kurth, Phys. Rev. D60 (1999) 014018.

13. J.A.M. Vermaseren, Int. J. Mod. Phys. A14 (1999) 2037.

14. J. Blümlein, Comput. Phys. Commun. 159 (2004) 19.

15. J. Blümlein, hep-ph/0402185

16. L. Euler, Novi Comm. Acad. Sci Petropolitanae 1 (1775) 140;

R.L. Graham, D.E. Knuth, and O. Patashnik, Concrete Mathematics, (Addison-Wesley, Reading/MA, 1994).

17. N. Nielsen, Handbuch der Theorie der Gammafunktion, (Teubner, Leipzig, 1906).

18. M. Hoffman, these Proceedings, math.QA/9907173

19. E. Carlson, Thesis, Univ. Uppsala, 1914, E.C. Titchmarsh, Theory of Functions, (Oxford University Press, Oxford, 1939), chap.9.5.

20. J. Blümlein and V. Ravindran, in preparation. 